\title{
PSEUDO-RIEMANNIAN MANIFOLDS WITH TOTALLY GEODESIC BISECTORS
}

\author{
JOHN K. BEEM
}

\begin{abstract}
Let $M$ be a pseudo-Riemannian manifold. Locally a distance function may be defined. The bisector of two points is the set of points equidistant from these two points. Our main result is that the bisector of two points which are not zero distance apart is a totally geodesic submanifold of $M$ if and only if $M$ has constant curvature.
\end{abstract}

1. Introduction. In a definite metric space the bisector of two points is the set of points equidistant from these two points. Blaschke [3] has shown that if $M$ is a two-dimensional Finsler space and satisfies the condition that the bisector of two points always lies on an extremal then $M$ is a Riemannian space of constant curvature. Busemann [4] has extensively studied $G$-spaces which have bisectors satisfying a flatness (geodesic) condition. In particular, Theorem 46.1 of [4] implies for Riemannian manifolds that a space has constant curvature if (locally) bisectors are totally geodesic submanifolds.

In this paper let $M$ denote a connected $n$-dimensional pseudo-Riemannian manifold with inner product $\langle$,$\rangle on the tangent space M_{p}$. A linear subspace $N_{p}$ of $M_{p}$ is nondegenerate if $\langle$,$\rangle is a nondegenerate inner prod-$ uct on $N_{p}$. We show that locally $M$ satisfies the requirement that the bisector of two points is a totally geodesic submanifold whenever the points are not zero distance apart if and only if $M$ has constant curvature.

2. The bisector condition. A result of Whitehead [7] implies that for each $r \in M$ there is a neighborhood $U(r)$ such that for all $p, q \in U(r)$ there is a unique extremal $\alpha(p, q)$ from $p$ to $q$ lying in $U(r)$. The set $U(r)$ is called a simple convex neighborhood of $r$. In $U(r)$ the separation between $p$ and $q$ is defined by

$$
d(p, q)=\int\langle\dot{x}, \dot{x}\rangle^{1 / 2} d t
$$

where $x(t)$ is a regular parametrization of $a(p, q)$. The number $d(p, q)$ is

Presented to the Society, January 23, 1975; received by the editors November 26, 1973 and, in revised form, March 20, 1974.

AMS (MOS) subject classifications (1970). Primary 53B 30. 
either real and nonnegative or is pure imaginary. In pseudo-Riemannian spaces the local distance function $d(p, q)$ is usually only defined for points sufficiently close together.

The bisector of $p$ and $q$ with respect to $U(r)$ is defined as

$$
B(p, q)=\left\{p^{\prime} \in U(r) \mid d\left(p, p^{\prime}\right)=d\left(p^{\prime}, q\right)\right\} .
$$

We will say $M$ satisfies the bisector condition if for each $r \in M$ there is a simple convex neighborhood $U(r)$ such that for all $p, q \in U(r)$ with $d(p, q)$ $\neq 0$ the bisector $B(p, q)$ is a totally geodesic submanifold of $U(r)$.

3. The two-dimensional case. If $n=2$, then the bisector condition is the requirement that $B(p, q)$ lie on a geodesic of $M$.

Proposition 1. Let $M$ be a two-dimensional pseudo-Riemannian manifold which satisfies the bisector condition. Then $M$ has constant curvature.

Proof. By [2, p. 1038] $M$ is a doubly timelike surface in the sense of [1]. Using the fact that $M$ is pseudo-Riemannian and Theorem (VI.36) of [1] it follows that $M$ has constant curvature.

4. The higher dimensional case. The next proposition is the pseudoRiemannian form of Theorem 24.15 of [6].

Proposition 2. Let $M$ be a pseudo-Riemannian manifold of dimension $n \geq 3$. Assume that each nondegenerate two-and three-dimensional tangent subspace is tangent to, respectively, a two- and three-dimensional totally geodesic submanifold. Then $M$ has constant curvature.

Proof. Let $N_{p}$ be a nondegenerate two-dimensional subspace of $M_{p}$. Applying the arguments of $\left[6\right.$, p. 340] we find $R\left(N_{p}, N_{p}\right)\left(N_{p}^{\perp}\right)=0$. Let $v_{1}, v_{2}$ be an orthonormal basis of $N_{p}$ and let $v$ be a unit vector in $N_{p}^{\perp}$. Then the skew-symmetric relation implies there are relations of the form

$$
\begin{array}{cc}
R\left(v_{1}, v_{2}\right)\left(v_{1}\right)=-a v_{2}, & R\left(v_{1}, v_{2}\right)\left(v_{2}\right)=e a v_{1}, \\
R\left(v_{1}, v\right)(v)=b v_{1}, & R\left(v_{1}, v\right)\left(v_{2}\right)=0,
\end{array}
$$

and $R\left(v_{1}, v_{2}\right)(v)=0$, where $e=\left\langle v_{1}, v_{1}\right\rangle\left\langle v_{2}, v_{2}\right\rangle$.

Let $\left.\left.e^{\prime}=\langle v, v\rangle\right\rangle_{2}, v_{2}\right\rangle$. If $e^{\prime}$ is positive then the arguments of [6, $\mathrm{p}$. 340] imply $b=e a=e^{\prime} e a$. On the other hand, if $e^{\prime}$ is negative then $v+1 / 2 v_{2}$ and $1 / 2 v+v_{2}$ are orthogonal. Hence,

$$
R\left(v_{1}, v+1 / 2 v_{2}\right)\left(1 / 2 v+v_{2}\right)=1 / 2(b+e a) v_{1}=0
$$

This yields $b=-e a=e^{\prime} e a$. 
This implies $M$ has constant sectional curvature at $p$, and by Corollary 2.2.7 of [8] we conclude $M$ has constant curvature.

Theorem 3. Let $M$ be a pseudo-Riemannian manifold. Then M has constant curvature if and only if $M$ satisfies the bisector condition.

Proof. First let $M$ satisfy the bisector condition. We may assume $n \geq$ 3 because of Proposition 1. Let $N_{p}$ be a nondegenerate $k$-dimensional linear subspace of $M_{p}$. By Proposition 2 it is sufficient to show $N_{p}$ is tangent to a $k$-dimensional totally geodesic submanifold. Let $N_{p}$ have orthonormal basis $v_{1}, \cdots, v_{k}$ and let $M_{p}$ have orthonormal basis $v_{1}, \cdots, v_{k}, \cdots, v_{n}$. Let $U(p)$ be a simple convex neighborhood of $p$ for which the bisector condition holds. Let $\exp ()$ be the exponential map at $p$. There is some $\delta>0$ such that $p_{i}=\exp \left(\delta v_{i}\right)$ and $q_{i}=\exp \left(-\delta v_{i}\right)$ lie in $U(p)$ for all $i=k+1$, $\cdots, n$. Then $\left|d\left(p_{i}, q_{i}\right)\right|=2 \delta$ and it is not hard to show $B\left(p_{i}, q_{i}\right)$ is an $(n-1)$-dimensional submanifold containing $p$. Furthermore, $v_{i}$ is orthogonal to the subspace of $M_{p}$ determined by $B\left(p_{i}, q_{i}\right)$.

Define $N=B\left(p_{k+1}, q_{k+1}\right) \cap \ldots \cap B\left(p_{n}, q_{n}\right)$. Then $N$ is totally geodesic and has tangent space $N_{p}$ at $p$.

Now let $M$ have constant curvature $K$ and $n \geq 2$. We wish to show that this implies $M$ satisfies the bisector condition. The notation will be the same as that in [8]. By Theorem 2.4.11 of [8] each $p \in M$ must have a neighborhood isometric to an open subset of $S_{s}^{n}$ if $K>0, R_{s}^{n}$ if $K=0$ and $H_{s}^{n}$ if $K<0$. If $K=0$, then the bisector condition is easily established.

If $K \neq 0$, then let $r \in \bar{M}$ where $\bar{M}=S_{s}^{n}$ if $K>0$ and $\bar{M}=H_{s}^{n}$ if $K<0$. Let $U(r)$ be a simple convex neighborhood in $\bar{M}$ of $r$. If $p, q \in U(r)$ with $d(p, q) \neq 0$, then let $p^{\prime}$ be the midpoint of $\alpha(p, q)$ and let $v$ be a unit tangent to $\alpha(p, q)$ at $p^{\prime}$. Let $P$ be the $n$-dimensional linear subspace of $R_{s}^{n+1}$ if $K>0$ or $R_{s+1}^{n+1}$ if $K<0$ such that $P$ contains $p^{\prime}$ and $P$ is orthogonal to $v$. Then $P \cap \bar{M}$ is a totally geodesic submanifold of $\bar{M}$ by Theorem 2.4.4 of [8]. The reflection in $P$ yields an isometry of $\bar{M}$ interchanging $p$ and $q$. Thus, the bisector $B(p, q)$ with respect to $U(r)$ contains $U(r) \cap P$. It still must be shown that $B(p, q)$ does not contain some point $q^{\prime} \notin U(r) \cap P$.

Jet $S_{p}$ ' be a two-dimensional linear subspace of $\bar{M}_{p}$ ' and let $S_{p}$ 'contain $v$. Then $\langle$,$\rangle restricted to S_{p}$ ' cannot be totally degenerate since $\langle v, v\rangle \neq 0$. Thus $\langle$,$\rangle either is nondegenerate on S_{p}{ }^{\prime}$ or is of rank 1 . Let $S$ be the submanifold of $U(r)$ determined by geodesics tangent to $S_{p}$ at $p^{\prime}$. It is not hard to show that $\langle$,$\rangle has the same rank on each tangent space to S$. Consider now some point $q^{\prime} \in B(p, q)$ with $q^{\prime} \notin U(r) \cap P$. Let $S$ be the 
two-dimensional totally geodesic submanifold of $U(r)$ containing $\alpha(p, q)$ and $q^{\prime}$. If $\langle$,$\rangle is positive definite or negative definite on S$ the triangle inequality gives a contradiction. If $\langle$,$\rangle is nondegenerate on S$ but not definite then the metric on $S$ makes $S$ a doubly timelike surface; see [2, p. 1038]. In this case there are two partial orderings $<_{1}$ and $<_{2}$ on $S$. If we have $m_{1}<_{i} m_{2}<{ }_{i} m_{3}$, then the reverse triangle inequality $\left|d\left(m_{1}, m_{2}\right)\right|+\left|d\left(m_{2}, m_{3}\right)\right|$ $\leq\left|d\left(m_{1}, m_{3}\right)\right|$ holds (compare [5, p. 244] and [1, p. 12]). There are several subcases to consider depending on how $p, q, q^{\prime}$ are related by the partial orderings. In each subcase the reverse triangle inequality gives a contradiction. If $\langle$,$\rangle has rank 1$ on $S$, then $S \cap B(p, q)$ is a single geodesic and $q^{\prime}$ cannot exist. This completes the proof.

\section{REFERENCES}

1. J. K. Beem and P. Y. Woo, Doubly timelike surfaces, Mem. Amer. Math. Soc. No. 92 (1969). MR $42 \# 1036$.

2. J. K. Beem, Indefinite Finsler spaces and timelike spaces, Canad. J. Math. 22 (1970), 1035-1039. MR $42 \# 2415$.

3. W. Blaschke, Geometrische Untersuchungen zur Variationsrechnung. I: Über Symmetralen, Math Z. 6 (1920), 281-285.

4. H. Busemann, The geometry of geodesics, Academic Press, New York, 1955. MR 17,779 .

5. H. Busemann and J. K. Beem, Axioms for indefinite metrics, Rend. Circ. Mat. Palermo (2) 15 (1966), 223-246. MR 38 \#1647.

6. R. Hermann, Differential geometry and the calculus of variations, Math. in Sci. and Engineering, vol. 49, Academic Press, New York, 1968. MR 38 \#1635.

7. J. H. C. Whitehead, Convex regions in the geometry of paths, Quart. J. Math. Oxford Ser. 3 (1932), 33-42. $36 \# 829$.

8. J. A. Wolf, Spaces of constant curvature, McGraw-Hill, New York, 1967. MR 65201

DEPARTMENT OF MATHEMATICS, UNIVERSITY OF MISSOURI, COLUMBIA, MISSOURI

Current address: Department of Mathematics, University of California, Berkeley, California 94720 